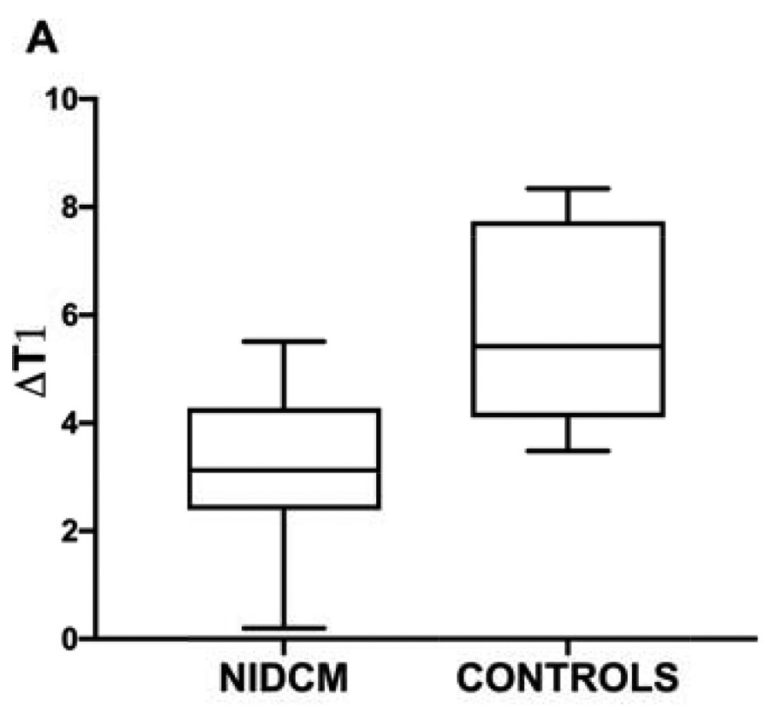

B

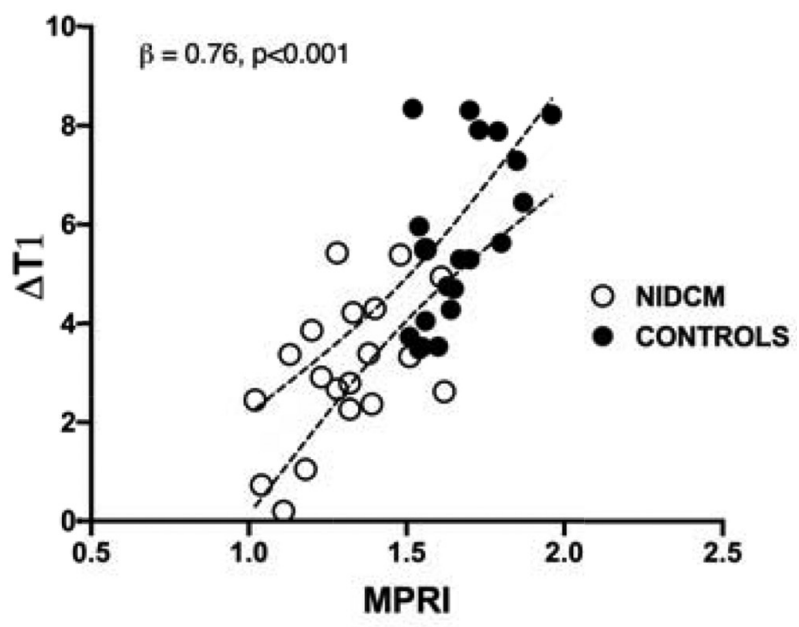

Abstract 24 Figure 1

ventricular slice for the assessment of rest and stress $\mathrm{T} 1$ values and $\mathrm{T} 1$ reactivity $(\Delta \mathrm{T} 1 \%) ; 3$. first-pass perfusion imaging for the assessment of myocardial perfusion reserve index (MPRI) and 4. late gadolinium enhancement (LGE) imaging for the assessment of myocardial replacement fibrosis, was performed. Twenty control patients without history of known coronary artery disease and evidence of reversible ischemia or previous myocardial infarct on CMR imaging were included for comparison purposes.

Results NIDCM patients had significantly higher native T1 value $(1263 \pm 47 \mathrm{~ms}$ vs. $1234 \pm 38 \mathrm{~ms}, \mathrm{p}=0.031)$, significantly lower $\Delta \mathrm{T} 1 \% \quad(3.2 \% \pm 1.5 \%$ vs. $5.7 \pm 1.7 \%, \quad \mathrm{p}<0.001$, figure $1 \mathrm{~A})$, significantly lower MPRI $(1.32 \pm 0.18$ vs. $1.67 \pm 0.13$, $\mathrm{p}<0.001)$ and significantly lower GLS $(-10 \pm 4 \%$ vs. -16 $\pm 2 \%, \mathrm{p}<0.001)$ as compared to controls. A significant strong relation between $\Delta \mathrm{T} 1 \%$ and MPRI $(B=0.76, p<0.001$, figure $1 \mathrm{~B})$ and a significant moderate relation between DT1\% and GLS $(ß=-0.54, \mathrm{p}<0.001)$ were observed.

Conclusion T1 reactivity, myocardial perfusion reserve and GLS are significantly reduced in NIDCM patients compared to controls. Adenosine stress T1 mapping holds promise for detection of impaired myocardial perfusion reserve in NIDCM without requirement for contrast agents.

\section{MITRAL INFLOW VELOCITY ENCODED IMAGING BY CMR FOR THE ASSESSMENT OF LEFT VENTRICULAR HAEMODYNAMICS}

${ }^{1}$ Nithin Balasubramanian, ${ }^{1}$ Natasha Barker, ${ }^{1}$ Benjamin Fidock, ${ }^{1}$ Alistair W Macdonald, ${ }^{1}$ David Capener, ${ }^{1}$ Christopher S Johns, ${ }^{2}$ Kavitasagary Karunasaagarar, ${ }^{2}$ Graham Fent,

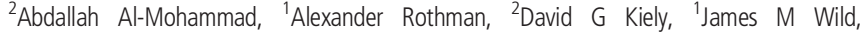
${ }^{1}$ Andrew Swift, ${ }^{1}$ Pankaj Garg. 'Department of Infection, Immunity and Cardiovascular disease, University of Sheffield, Sheffield, UK; ${ }^{2}$ Sheffield Teaching Hospitals NHS Foundation Trust, Sheffield, UK

\subsection{6/heartjnl-2019-BSCMR.25}

Background Heart failure is common and is associated with high mortality and morbidity. Haemodynamic assessment is critical to diagnosis and therapeutic monitoring. Non-invasive Doppler echocardiography is commonly used to assess peak early and late mitral inflow velocities to predict left ventricular (LV) haemodynamics. Cardiovascular magnetic resonance imaging offers two-dimensional through plane flow assessment using phase contrast acquisition. It remains unclear which through plane mitral inflow velocity (averaged or peak) best predicts LV haemodynamics better.

Purpose The objective of this study is to investigate which through plane mitral inflow velocities (averaged or peak) is better associated with invasive left heart haemodynamic parameters. Methods In this observational study, 16 patients were prospectively recruited at a large tertiary pulmonary hypertension unit. These patients had invasive right heart haemodynamic catheter-based study (RHC). In addition, all patients received CMR on the $1.5 \mathrm{~T}$ scanner (HDx scanner, GE Healthcare, Waukesha, Wisconsin, USA), using an 8-channel cardiac coil. CMR protocol included long and short axis cines and mitral inflow phase contrast acquisition. The LV haemodynamic parameters assessed by RHC included the following: pulmonary capillary wedge pressure (PCWP), cardiac output, cardiac index, systemic vascular resistance (SVR). Cardiac output assessment was done by standard thermodilution method.

Results Mean age of the 16 patients analysed was $68.7 \pm 7.9$ years and $56.3 \%$ were female. Of the 16 patients, 5 patients had non-analysable data. Mean PCWP was $11.1 \pm 4.7 \mathrm{mmHg}$, mean cardiac output was $4.4 \pm 1.3 \mathrm{~L} / \mathrm{min}$, mean cardiac index was $2.4 \pm 0.8 \mathrm{~L} / \mathrm{min} / \mathrm{m}^{2}$ and mean SVR was $1827 \pm 589$ dynes/ seconds $/ \mathrm{cm}^{-5}$. Peak EA ratio correlated with PCWP $(r=0.68$ $\mathrm{p}=0.02)$ and with cardiac index $(\mathrm{r}=0.68 \mathrm{p}=0.02)$. There was a notable correlation between mean EA ratio and cardiac index $(\mathrm{r}=0.66 \mathrm{p}=0.03)$. Mean A showed a significant correlation with SVR $(r=0.64, p=0.03)$.

Conclusions Peak mitral inflow velocities are more closely associated with LV haemodynamics. This may be due to

Abstract 25 Table 1 Mitral inflow 2D flow parameters and their association to left heart haemodynamics

\begin{tabular}{lccccc}
\hline CMR parameters & Mean & SD & \multicolumn{3}{c}{ Pearson Correlation (P-value) } \\
\hline & & & PCWP & Cardiac Index & SVR \\
\hline Peak E velocity (cm/sec) & 55.6 & 29 & $0.41(0.13)$ & $0.22(0.33)$ & $0.28(0.53)$ \\
Peak A velocity (cm/sec) & 52.2 & 19 & $0.30(0.37)$ & $0.24(0.17)$ & $0.37(0.06)$ \\
EA ratio & 1.1 & 0.5 & $0.69(0.02)$ & $0.68(0.02)$ & $0.58(0.07)$ \\
MV DT (milliseconds) & 210 & 71 & $0.32(0.10)$ & $0.29(0.44)$ & $0.34(0.55)$ \\
Mean E (cm/sec) & 13.4 & 6 & $0.21(0.55)$ & $0.14(0.22)$ & $0.17(0.90)$ \\
Mean A (cm/sec) & 8.2 & 4 & $0.44(0.17)$ & $0.56(0.03)$ & $0.64(0.03)$ \\
Mean EA ratio & 2.2 & 2 & $0.41(0.21)$ & $0.66(0.03)$ & $0.44(0.20)$ \\
\hline
\end{tabular}



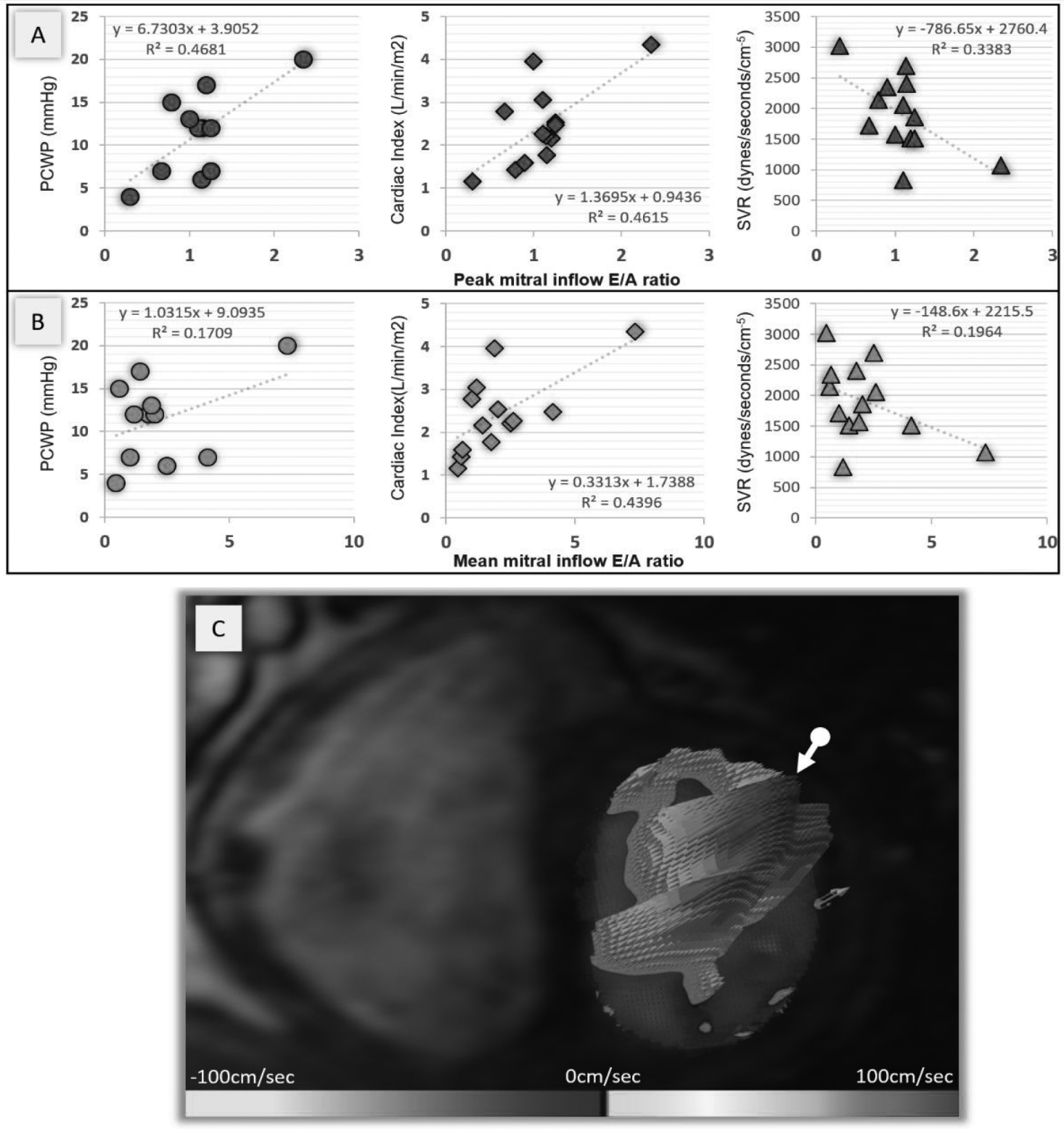

Abstract 25 Figure 1 Panel A: Scatter plots of invasive haemodynamic parameters to peak mitral inflow parameters. Panel B: Scatter plots of invasive haemodynamic parameters to mean mitral inflow parameters. Panel C: Phase contrast image overlay demonstrating signal intensity change of velocity through the mitral valve. The mean velocity is the average through the whole mitral valve and the peak is highlighted using the white arrow

reduced variation in through-plane peak velocity at different inflow levels versus the mean velocity. Future work is required to determine the effect of addressing the valvular throughplane motion, which may make the averaged mitral inflow assessment a better tool for LV hemodynamics.

\section{A NON-INVASIVE CMR ASSESSMENT FOR PREDICTING MEAN PULMONARY ARTERY PRESSURE IN PULMONARY HYPERTENSION}

${ }^{1}$ Benjamin Fidock, ${ }^{1}$ Nithin Balasubramanian, ${ }^{1}$ Natasha Barker, ${ }^{1}$ Alistair W Macdonald, ${ }^{1}$ David Capener, ${ }^{1}$ Christopher $S$ Johns, ${ }^{2}$ Kavitasagary Karunasaagarar, ${ }^{2}$ Graham Fent, ${ }^{2}$ Abdallah Al-Mohammad, 'Alexander Rothman, ${ }^{2}$ David G Kiely, 'Andrew Swift, 'James M Wild, 'Pankaj Garg. 'Department of Infection, Immunity and Cardiovascular Disease, University of Sheffield, Sheffield, UK; ${ }^{2}$ Sheffield Teaching Hospitals NHS Foundation Trust, Sheffield, UK

10.1136/heartjnl-2019-BSCMR.26
Background Pulmonary hypertension (PH) is defined by a resting mean pulmonary artery pressure (mPAP) $\geq 25 \mathrm{mmHg}$ by right heart catheterization. Systolic pulmonary artery pressures are used as surrogate non-invasive markers of $\mathrm{PH}$. A direct prediction tool of non-invasive mPAP would provide the capacity to diagnose disease through non-invasive means. It remains unclear if a multi-parametric cardiac magnetic resonance (CMR) integrated approach can provide an accurate measure of invasive mPAP.

Purpose This study sought to develop a novel CMR model using both established and newly derived CMR metrics for estimating mean pulmonary artery pressure.

Methods 18 patients were prospectively recruited at a large tertiary $\mathrm{PH}$ unit. All patients underwent right heart catheterisation (RHC) and CMR on the $1.5 \mathrm{~T}$ scanner (HDx scanner, GE Healthcare, Waukesha, Wisconsin, USA), using an 8-channel cardiac coil. CMR protocol included long and short axis cines and through-plane pulmonary artery phase contrast 